\title{
Stimulus-Specific Adaptation: Can It Be a Neural Correlate of Behavioral Habituation?
}

\author{
Shai Netser, Yael Zahar, and Yoram Gutfreund \\ The Department of Physiology and Biophysics, The Ruth \& Bruce Rappaport Faculty of Medicine, Technion, Haifa 31096, Israel
}

Habituation is the most basic form of learning, yet many gaps remain in our understanding of its underlying neural mechanisms. We demonstrate that in the owl's optic tectum (OT), a single, low-level, relatively short auditory stimulus is sufficient to induce a significant reduction in the neural response to a stimulus presented up to $60 \mathrm{~s}$ later. This type of neural adaptation was absent in neurons from the central nucleus of the inferior colliculus and from the auditory thalamus; however, it was apparent in the 0T and the forebrain entopallium. By presenting sequences that alternate between two different auditory stimuli, we show that this long-lasting adaptation is stimulus specific. The response to an odd stimulus in the sequence was not smaller than the response to the same stimulus when it was first in the sequence. Finally, we measured the habituation of reflexive eye movements and show that the behavioral habituation is correlated with the neural adaptation. The finding of a long-lasting specific adaptation in areas related to the gaze control system and not elsewhere suggests its involvement in habituation processes and opens new directions for research on mechanisms of habituation.

\section{Introduction}

Habituation is defined as behavioral response decrement that results from repeated stimulation and does not involve response fatigue (Thompson and Spencer, 1966). The distinction between fatigue and habituation is commonly made by demonstrating that the response decrement is specific to the repeated stimulus (Barry, 2009; Rankin et al., 2009). Thus, habituation is a learning process that allows the animal to ignore irrelevant stimuli and focus on novel important stimuli. Habituation is considered the most basic form of learning that exists in all animals and all senses (Thompson, 2009). Despite its importance, many gaps remain in our understanding of the neural mechanisms of habituation (Rankin et al., 2009).

At the neural level, stimulus-specific adaptation (SSA) is a phenomenon that resembles behavioral habituation in several aspects. First, in SSA, the response of a neuron to a stimulus is decreased when the same stimulus is presented repeatedly. But more importantly, the decrease is specific to the stimulus; changing the stimulus (frequency, amplitude, location, etc.) regains the neural response (Sobotka and Ringo, 1994; Ulanovsky et al., 2003; Katz et al., 2006; Reches and Gutfreund, 2008). This phenomenon has recently attracted scientific interest, particularly in the auditory system (Nelken and Ulanovsky, 2007; Anderson et al., 2009; Malmierca et al., 2009; Farley et al., 2010). It has been suggested to play a role in auditory scene analysis, optimal coding, and novelty detection (Ulanovsky et al., 2004). But can it be a neural

Received Sept. 20, 2011; revised 0ct. 16, 2011; accepted 0ct. 21, 2011.

Author contributions: S.N. and Y.G. designed research; S.N. performed research; S.N. and Y.Z. analyzed data; S.N. and Y.G. wrote the paper.

This work was supported by a grant from the Israel Science Foundation. We thank Prof. Shimon Marom for critical reading of the manuscript.

Correspondence should be addressed to Yoram Gutfreund, Department of Physiology and Biophysics, The Bruce Rappaport Medical School, The Technion, Haifa 31096, Israel. E-mail: yoramg@tx.technion.ac.il.

DOI:10.1523/JNEUROSCI.4790-11.2011

Copyright $\odot 2011$ the authors $\quad 0270-6474 / 11 / 3117811-10 \$ 15.00 / 0$ correlate of behavioral habituation to an auditory stimulus? One major caveat of this hypothesis is the different time scales. Most forms of SSA were demonstrated and studied using interstimulus intervals (ISIs) of up to $2 \mathrm{~s}$ (Ulanovsky et al., 2003; Nelken and Ulanovsky, 2007; Reches and Gutfreund, 2008; Anderson et al., 2009; Malmierca et al., 2009; Antunes et al., 2010; Farley et al., 2010). Behavioral habituation, on the other hand, acts at ISIs of tens of seconds to minutes, even for relatively short-duration stimuli (Weinberger et al., 1975; Valentinuzzi and Ferrari, 1997; Bala and Takahashi, 2000; Zimmer, 2006; Dong and Clayton, 2009). It is therefore necessary to identify a form of neural adaptation that maintains a comparable memory trace.

For this purpose, we recorded single-unit and multiunit responses of neurons from the optic tectum (OT, a midbrain gaze control center that is homologous to the superior colliculus of mammals). We report a new form of neural adaptation that has a relatively long memory trace (up to a minute and possibly more) and is stimulus specific. We further show that this form of adaptation is absent in the ascending auditory pathway to the OT and in the primary auditory thalamus, but is apparent, in addition to the OT, in the entopallium (E), a forebrain area connected to the gaze control system (Benowitz and Karten, 1976; Reches and Gutfreund, 2009). We therefore propose that gaze control circuitry, which is involved in the selection of salient stimuli in space (Boehnke and Munoz, 2008; Mysore et al., 2010), is also involved in the selection of salient stimuli in time.

\section{Materials and Methods}

Experimental animals. A total of 15 barn owls (Tyto alba) of either sex were used in this study. All owls were hatched in captivity and raised and kept in a large flying cage equipped with perching spots and nesting boxes. The owls were provided for in accordance with guidelines of the Technion Institutional Animal Care and Use Committee.

Electrophysiological procedures. The birds were prepared for repeated experiments in a single surgical procedure: the birds were anesthetized 


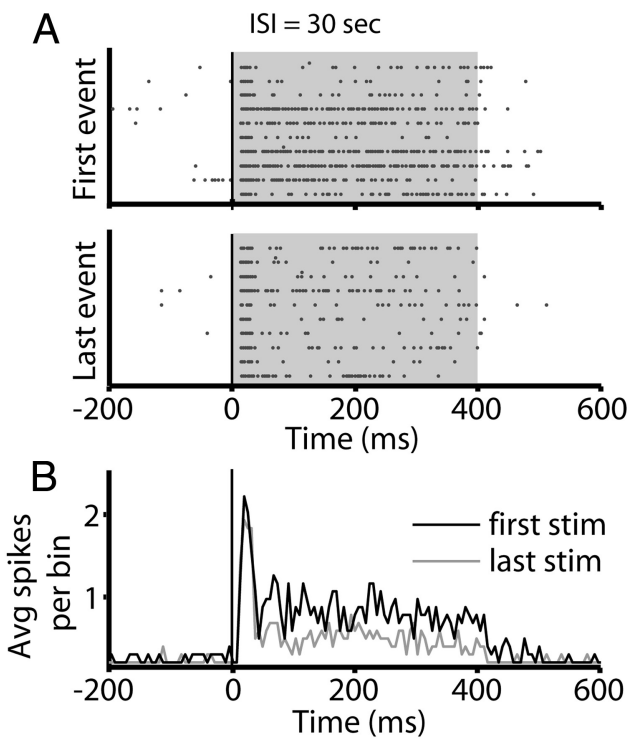

Figure 1. A single-site example of adaptation to a repeated auditory stimulus with an ISI of $30 \mathrm{~s}$. $A$, The raster plot in the upper panel shows responses to the first stimulus in the sequence. The raster plot in the lower panel shows responses to the last stimulus in the sequence. The gray area represents the stimulus duration. $\boldsymbol{B}$, The PSTH of the responses to the first stimulus (black curve) compared to the PSTH of the responses to the last stimulus (gray curve). Auditory stimulus was broadband ( $3-10 \mathrm{kHz}$ ) and $400 \mathrm{~ms}$ long with an ITD of $-3 \mu \mathrm{s}$ and ILD of $-1 \mathrm{~dB}$.

with $2 \%$ isoflurane in a mixture of nitrous oxide and oxygen (4:5). A craniotomy was performed and a recording chamber was cemented to the skull. At the beginning of each electrophysiological session, the owl was anesthetized briefly with isoflurane $(2 \%)$ and nitrous oxide in oxygen (4:5). Once anesthetized, the animal was positioned in a stereotaxic apparatus at the center of a double-wall, sound-attenuating booth. The head was fixed and aligned using the pecten oculi as a retinal landmark (Wathey and Pettigrew, 1989). Within the booth, isoflurane was removed and the bird was maintained on a fixed mixture of nitrous oxide and oxygen (4:5). The cover of the craniotomy was removed and a platinum-iridium or tungsten, epoxy-coated electrode (0.5-2 M $\Omega$ ) was driven into the recording chamber. A Tucker-Davis Technologies System 3 and an online spike sorter (MSD, Alpha-Omega) were used to isolate action potentials from a small cluster of neurons (multiunit recording) or single neurons (single-unit recording). Single units were isolated using template-based sorting. At the end of each recording session, the recording chamber was treated with chloramphenicol ointment (5\%) and closed. The owl was then returned to its home flying cage.

Targeting of nuclei. Identification of the location of the recording site was based on stereotaxic coordinates and on the expected physiological properties of each nucleus: the OT was recognized by characteristic bursting activity and spatially restricted visual and auditory receptive fields. Position within the OT was determined based on the location of the visual receptive field (RF). The deep layers of the OT were located beneath the bursty layers and identified based on the characteristic regular firing. The external nucleus of the inferior colliculus (ICX) was targeted by positioning the electrode $2 \mathrm{~mm}$ caudal and $2.5 \mathrm{~mm}$ medial from the tectal representation of $0^{\circ}$ azimuth and $+10^{\circ}$ elevation relative to the visual axes (tectal reference point). The ICX was identified by broadband frequency tunings and robust mapping of interaural time and level differences (ITD and ILD, respectively). The central nucleus of the inferior colliculus (ICC) was targeted $3.5 \mathrm{~mm}$ medial and $3 \mathrm{~mm}$ caudal to the same tectal reference point. The ICC was identified by narrowband frequency tunings and tonotopic mapping. The nucleus ovoidalis (nOv) was targeted $1.2 \mathrm{~mm}$ rostral and $5 \mathrm{~mm}$ medial from the tectal representation of $0^{\circ}$ azimuth and $0^{\circ}$ elevation relative to the visual axes. In $\mathrm{nOv}$, we searched for robust auditory responses to binaural stimulation, high levels of spontaneous activity, and tonotopic mapping. The E was targeted $2 \mathrm{~mm}$ rostral, $0.4 \mathrm{~mm}$ lateral, and $6.5-7 \mathrm{~mm}$ dorsal from the same
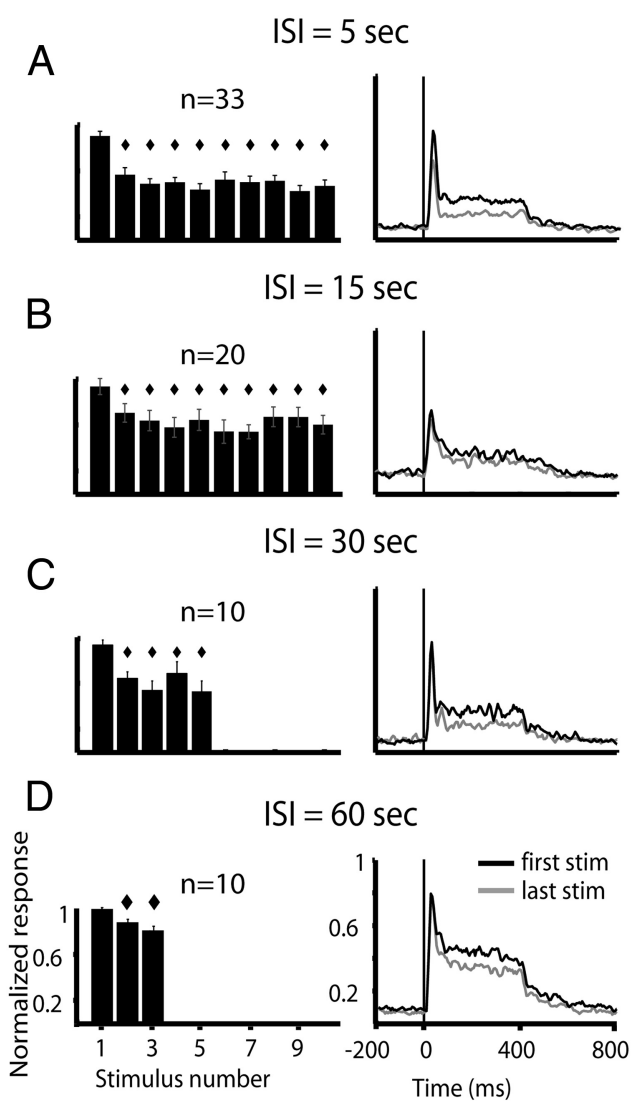

Figure 2. Neural adaptation in the OT with ISIs of 5-60 s. A, Population response to stimulation with an ISI of $5 \mathrm{~s}$. The histogram on the left shows the population response to an auditory stimulus as a function of its position in the sequence. Error bars designate SEMs. Diamonds designate responses that were significantly smaller than the response to the first stimulus in the sequence ( $t$ test, Holm-Bonferroni correction, $p<0.05$ ). The number above the histogram indicates the number of recording sites. On the right, the population PSTH of the response to the first stimulus (black curve) is compared to the population PSTH of the response to the last stimulus in the sequence ( 45 s later, gray curve). $\boldsymbol{B}$, Population response to stimulation with an ISI of $15 \mathrm{~s}$. The histogram on the left shows the population response to the stimulus as a function of its position in the sequence. On the right, the population PSTH of the response to the first stimulus (black curve) is compared to the population PSTH of the response to the last stimulus in the sequence ( 135 s later, gray curve). The format is as in $\boldsymbol{A}$. C, Population response to stimulation with an ISI of $30 \mathrm{~s}$. The histogram on the left shows the population response to the stimulus as a function of its position in the sequence. On the right, the population PSTH of the response to the first stimulus (black curve) is compared to the population PSTH of the response to the last stimulus in the sequence ( 120 s later, gray curve). The format is as in $\boldsymbol{A}$. D, Population response to stimulation with an ISI of $60 \mathrm{~s}$. The histogram on the left shows the population response to the stimulus as a function of its position in the sequence. $0 \mathrm{n}$ the right, the population PSTH of the response to the first stimulus (black curve) is compared to the population PSTH of the response to the last stimulus in the sequence (120 s later, gray curve). The format is as in $\boldsymbol{A}$.

tectal reference point. In the E, we recorded in the area in which we have previously identified auditory responses (Reches and Gutfreund, 2009). This area was characterized by a high level of spontaneous bursty activity and strong responses to visual stimuli. The correspondence of the stereotaxic coordinates and the characteristic physiological responses with the desired brain locations were previously confirmed with anatomical reconstructions of the recording sites (Knudsen, 1982; Wagner et al., 1987; Brainard and Knudsen, 1993; Gutfreund et al., 2002; Miller and Knudsen, 2003; Pérez and Peña, 2006; Reches and Gutfreund, 2009; Netser et al., 2010). In all nuclei, the electrode was advanced within the nucleus in $300 \mu \mathrm{m}$ steps; at each step, basic auditory responses (frequency, ITD, ILD) and visual responsiveness were first measured to make sure that the recording site is within the desired nucleus. All recording sites that were characterized to be within the nucleus were included in the analysis. 

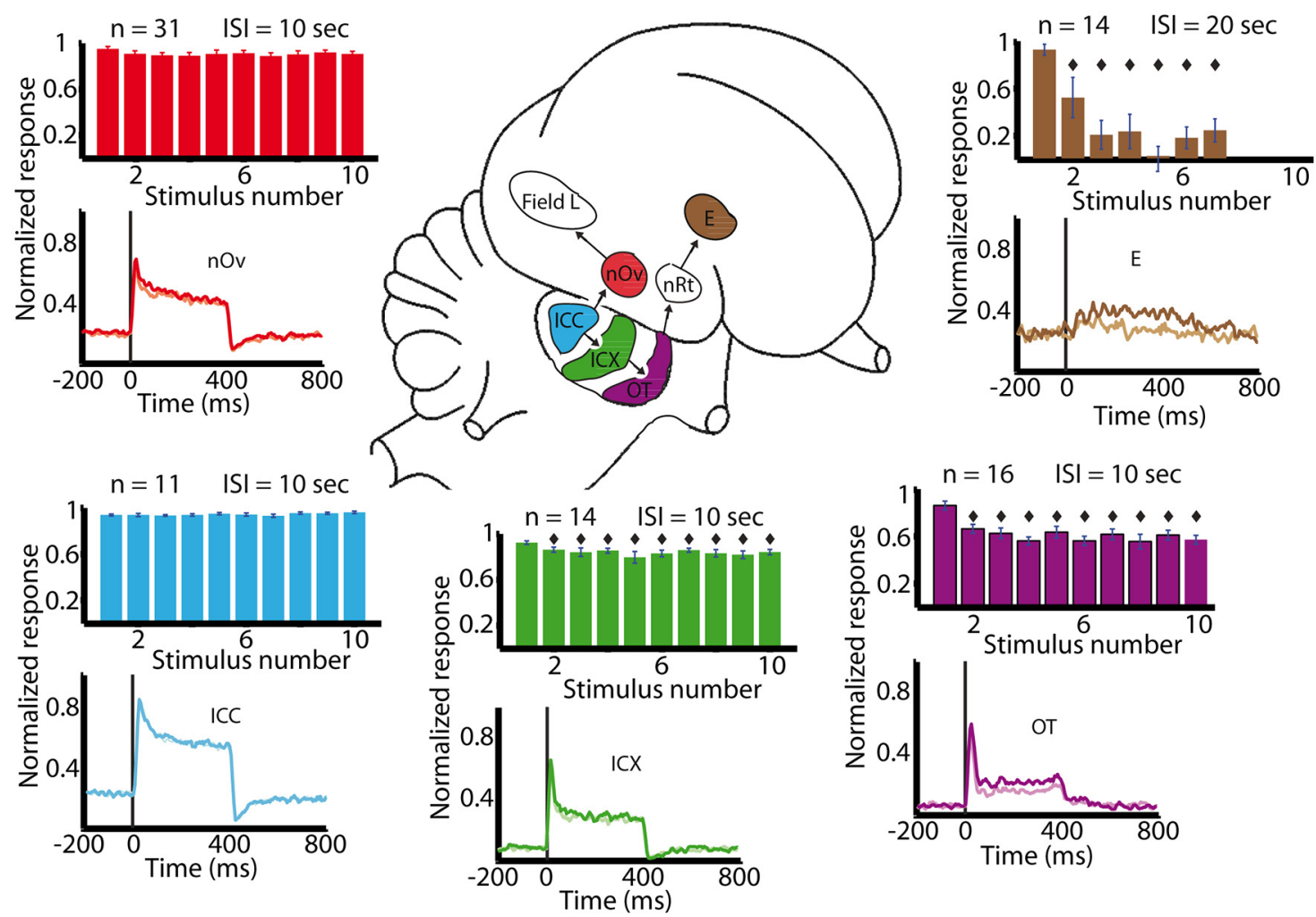

Figure 3. Neural adaptation in different brain nuclei. The inset shows a scheme of a lateral view of the barn owl's brain. Seven nuclei are marked in the scheme. The arrows indicate the typical direction of information flow. The histograms show the population response, in each of the nuclei examined, as a function of the position of the stimulus in the sequence. Below each histogram, the population PSTH of the response to the first stimulus (dark curve) is compared to the population PSTH of the response to the last stimulus in the sequence (light curve). The colors of the bars and curves correspond to the colors of the corresponding nuclei in the inset. The error bars designate SEMs. The diamonds indicate responses that were significantly smaller from the response to the first stimulus in the sequence ( $t$ test, Holm-Bonferroni correction; $p<0.05$ ). nRt, Nucleus rotundus.

Auditory stimulation. Sounds for stimulation were generated on a computer and emitted through miniature earphones (Knowles ED1914). The earphones were placed in the center of the ear canal $\sim 8 \mathrm{~mm}$ from the tympanic membrane. The amplitude and phase spectra of the earphones were equalized within $\pm 2 \mathrm{~dB}$ and $\pm 2 \mu$ s between 2 and 12 $\mathrm{kHz}$. Sound levels were controlled by two independent attenuators (TDT PA5).

Tuning curves were generated by varying a single parameter (ITD, ILD, central frequency, or sound intensity) while holding all other parameters constant. Sounds for tuning curves measurements were broadband $(3-10 \mathrm{kHz})$ or narrowband $(1 \mathrm{kHz}$ width), were $100 \mathrm{~ms}$ long, and had a rise/fall time of $5 \mathrm{~ms}$. The value of the tested parameter was varied randomly in stimulus sets that were repeated 10 times.

To test adaptation, we used, depending on the paradigm, broadband sounds (3-10 kHz, $400 \mathrm{~ms}$ duration, $5 \mathrm{~ms}$ rise/fall time), narrowband sounds ( $1 \mathrm{kHz}$ width, $400 \mathrm{~ms}$ duration, $5 \mathrm{~ms}$ rise/fall time), or recordings of natural sounds. Natural sounds were chosen from a library of six possible sounds emitted by mice, rats, or owls (see spectrograms and waveforms in Fig. 6). The natural sounds were truncated with $5 \mathrm{~ms}$ rise and fall times to a duration of $300 \mathrm{~ms}$. Sound levels of both computersynthesized and natural sounds were adjusted to $\sim 20 \mathrm{~dB}$ above the site's response threshold (15-30 dB SPL). In all cases except for the E, the sounds were presented at the unit's best ITD and ILD, determined separately for each recording site (see below, Data analysis). E neurons have large contralateral RFs with a slight preference for negative ILDs (Reches and Gutfreund, 2009). Thus, in these experiments the best values were not determined and the ITDs of the sounds were chosen between 40 and $100 \mu$ s contralateral ear-leading and the ILDs between 0 and $-6 \mathrm{~dB}$ (left ear louder). The adaptation was tested by presenting the sounds in regular sequences whose interstimulus intervals (ISIs) and number of stimuli differed depending on the paradigm tested. Each sequence was repeated 11 times. Intersequence intervals (time between the last stimu- lus in the sequence and the first stimulus in the next sequence) were 1 min and $10 \mathrm{~s}$ for the $10 \mathrm{~s}$ ISI tests, $1 \mathrm{~min}$ and $15 \mathrm{~s}$ for the $15 \mathrm{~s}$ ISI tests, 2.5 min for the $30 \mathrm{~s}$ ISI tests, and $5 \mathrm{~min}$ for the $60 \mathrm{~s}$ ISI test.

Tracking eye movements. Eye movements were measured while the owls were positioned in the electrophysiological set-up described above, breathing a mixture of 4:5 $\mathrm{N}_{2} \mathrm{O}$ /oxygen. The method to evaluate eye movements is described in detail by Netser et al. (2010). Briefly, the owl's right eye was held open by attaching miniature clips to the small feathers on the eyelids. The clips were gently pulled by strings to adjust a sufficient and stable retraction of the eyelid. An infrared (IR)-sensitive digital video camera equipped with a zoom lens was positioned $\sim 1 \mathrm{~m}$ from the owl's head. An IR LED was attached to the camera lens and adjusted manually in each session to optimally capture the IR light reflected from the retina. The camera angle relative to the head was then adjusted so that the lateral edge of the pecten oculi was visible at the center of the pupil (see inset in Fig. 8).The pecten oculi is an apparent retinal landmark (Wathey and Pettigrew, 1989). Once the camera was positioned in place, the door of the booth was closed and video sequences ( $30 \mathrm{fps}$ ) were collected starting $500 \mathrm{~ms}$ before the onset of the stimulus and ending $3 \mathrm{~s}$ after. Video sequences were analyzed offline. Trials containing eye blinks, nictitating membrane movements, or vigorous pecten movements during the prestimulus time window (500 ms) were discarded from the analysis.

Data analysis. Neural responses to an acoustic stimulus were quantified as the number of spikes in a given time window after stimulus onset minus the number of spikes during the same amount of time immediately before stimulus onset (baseline activity). The duration of the time window for spike count corresponded to the duration of the stimulus. The width of tuning curves was defined as the range over which responses were $>50 \%$ of the maximal response; best ITD, ILD, or frequency was the midpoint of this range.

To observe the time course of the response, we generated poststimulus time histograms (PSTHs) with $8 \mathrm{~ms}$ time bins. PSTHs were normalized 
to the maximum bin and averaged across the population. The averaged population PSTHs (Figs. 2-5, 7) were smoothed for display purposes (running average of 3 points).

To analyze the adaptation tests, we first averaged the responses to each basic sequence across sequence repetitions, yielding the average response to a stimulus as a function of the stimulus position in the sequence. The first sequence in each test was omitted from the analysis to avoid possible biases that may result from the first sequence being novel.

To obtain the population response, we normalized the responses from each site to its maximum response and averaged across all recording sites. To test the significance of adaptation, we performed one-tailed $t$ tests to compare the population average response of the first stimulus in the sequence with the average responses to all the remaining stimuli. A HolmBonferroni multiple-comparison correction (Holm, 1979) was applied to all tests.

To quantify the SSA effect, we calculated the stimulus index (SI), which is the normalized difference between the responses to the novel appearance and the frequent appearance defined as follows:

$$
S I_{1}=\frac{N_{1}-F_{1}}{N_{1}+F_{1}} ; S I_{2}=\frac{N_{2}-F_{2}}{N_{2}+F_{2}}
$$

where $N_{1}$ is the response to the first (novel) stimulus in the sequence of stimulus $1, F_{1}$ is the average response to the last three stimuli in the sequence of stimulus $1, N_{2}$ is the response to the first (novel) stimulus in the sequence of stimulus 2 , and $F_{2}$ is the average response to the last three stimuli in the sequence of stimulus 2 .

To quantify the tendency of the neuron to respond to a rare stimulus independent of the stimulus itself, we used the neuron index (NI), defined as follows:

$$
N I=\frac{\left(N_{1}-F_{1}\right)+\left(N_{2}-F_{2}\right)}{N_{1}+F_{1}+N_{2}+F_{2}}
$$

To quantify the eye movements, we first converted the grayscale video images to binary images. In each video frame, we counted the number of white pixels in a manually defined rectangle that overlapped the pecten edge. This produced a response curve corresponding to the horizontal position of the pecten edge as a function of time (see detailed description and evaluation of the method by Netser et al., 2010). Then the baseline was reduced from the response curve [i.e., subtracting the average pecten position in the prestimulus interval $(-500$ to $0 \mathrm{~ms})]$. The eye response magnitude was defined as the sum of the pecten position values during the poststimulus time window (i.e., area under the response curve between 0 and $600 \mathrm{~ms}$ ). To obtain the population response, we normalized the responses from each experiment to its maximal response and averaged across all experiments. To test the significance of habituation, we performed one-tailed $t$ tests to compare the population average response of the first stimulus in the sequence with the average responses to all the remaining stimuli. A Holm-Bonferroni multiple-comparison correction was applied to all tests.

\section{Results}

\section{Long-lasting adaptation in the OT}

We recorded the responses of neurons in the deep and intermediate layers of the OT. Each recording site was tested with sequences of identical auditory stimuli at its best ITD and ILD (see Materials and Methods). The ISIs of the sequences were 5, 15, 30, or $60 \mathrm{~s}$. Figure 1 shows an example of a multiunit recording from a single tectal site. In this example, sequences of five auditory stimuli with an ISI of $30 \mathrm{~s}$ were presented (2.5 min gap between sequences). It is evident that this recording site tended to respond stronger to the first stimulus in the sequence (upper raster plot) compared to the last (lower raster plot). The tendency to respond less to subsequent stimuli was observed at the population level. The histograms in Figure 2 show the population responses to sequences with different ISIs. The population responses to the first stimuli were significantly larger ( $t$ test with Holm-Bonfer-
A
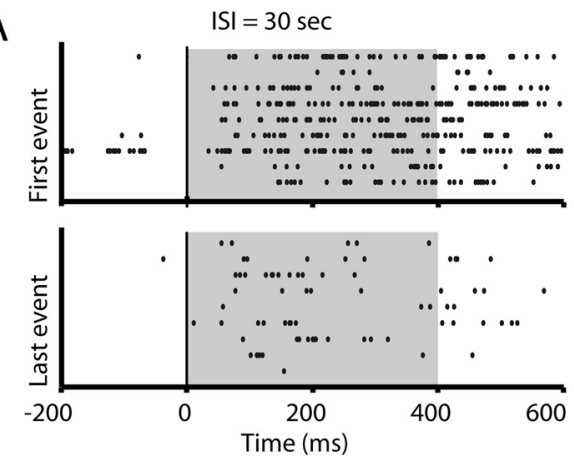

B
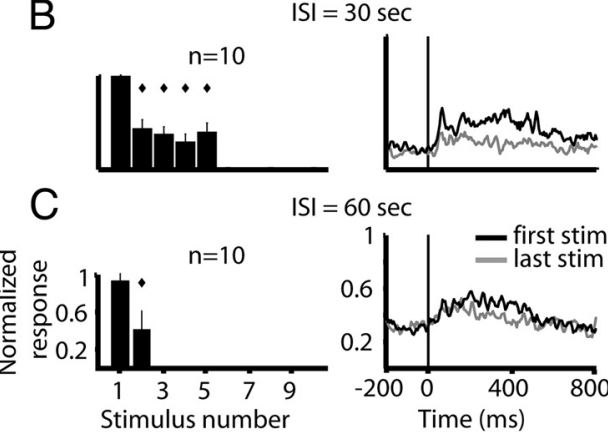

Figure 4. Neural adaptation in the $E$ with ISIs of 30 and 60 s. A, A single-site example of adaptation in the $E$ to a repeated auditory stimulus with an ISI of $30 \mathrm{~s}$. The raster plot in the upper panel shows responses to the first stimulus in the sequence. The raster plot in the lower panel shows responses to the last (fifth) stimulus in the sequence. The gray area represents the stimulus duration. $\boldsymbol{B}$, Population responses in the E to sequences with an ISI of $30 \mathrm{~s}$. The format is as in Figure 2A.C, Population responses in the E to sequences with an ISI of $60 \mathrm{~s}$. The format is as in Figure $2 A$.

roni correction, $p<0.05)$ than the responses to all subsequent stimuli at all ISIs tested (Fig. $2 A-D$ ). A single, relatively short (400 ms), close-to-threshold stimulus (see Materials and Methods) was sufficient to induce a significant reduction of the response to a second stimulus presented up to 1 min later (Fig. 2D). Therefore, this neural adaptation was strong, rapid, and long lasting.

\section{Long-lasting adaptation outside the OT}

To track the origin of the long-lasting adaptation, we recorded from five nuclei along the auditory pathways: the ICC, the ICX, the OT, the E, and the nOv. In each nucleus, multiple recording sites were isolated (see Materials and Methods) and tested with a simple adaptation test (11 repetitions of the same broadband sound with an ISI of $10 \mathrm{~s}$ ). The average responses are shown in the histograms in Figure 3. The neural responses in the ICC, which is the source of the auditory inputs to both the primary auditory pathway (thalamofugal pathway) and the midbrain localization pathway (Arthur, 2005), did not show a tendency for adaptation at $10 \mathrm{~s}$ ISIs (blue histogram, $n=11$ ). This observation suggests that the long-lasting adaptation reported in the OT develops at higher levels of the auditory pathway. Long-lasting adaptation was also not observed in nOv (red histogram, $n=31$ ), the primary thalamic auditory nucleus. This was in sharp contrast to the results from the OT and the E, which showed clear neural adaptations at long ISIs (purple and brown histograms, $n=16$ and $n=14$, respectively). The $\mathrm{E}$ is the forebrain recipient of the tectofugal pathway (Benowitz and Karten, 1976), which in barn owls carries auditory as well as visual signals (Reches and Gutfreund, 2009). The auditory responses in the E were slower and 


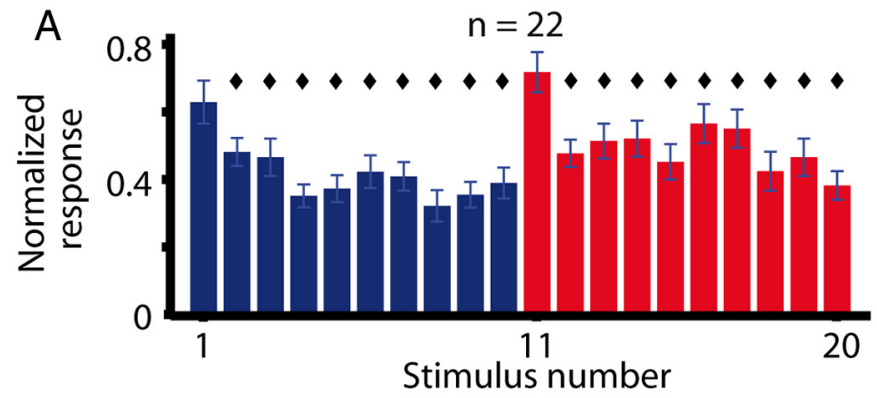

B

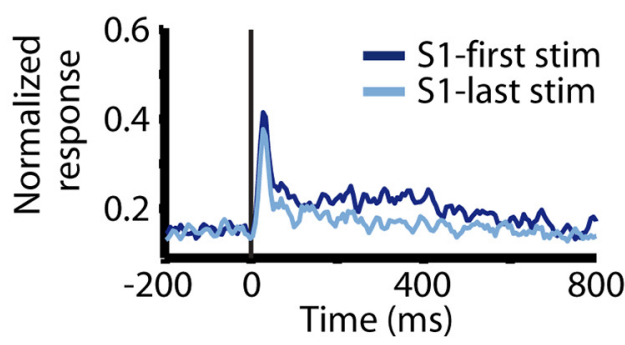

D

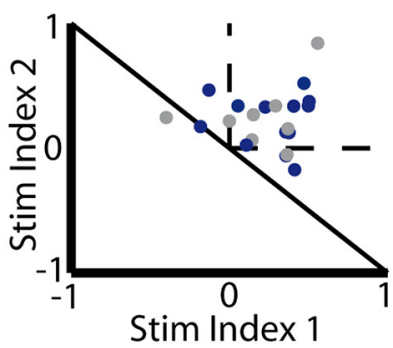

C
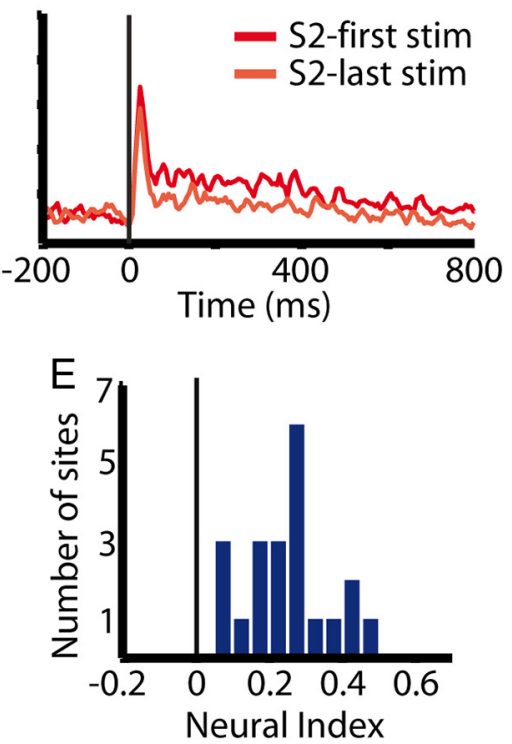

Figure 5. Long-lasting adaptation in the OT is stimulus specific. $\boldsymbol{A}$, The population response as a function of the position of the stimulus in the sequence $(I S I=10 \mathrm{~s}$ ). The blue bars (positions $1-10$ ) designate the responses to the 10 repetitions of stimulus 1 and the red bars (positions 11-20) to the 10 repetitions of stimulus 2 . The two stimuli differ by their central frequency $(\Delta F=2 \mathrm{kHz})$. Error bars indicate SEMs. Diamonds represent responses that were significantly lower than the response to the same stimulus when it was first in its sequence ( $t$ test, Holm-Bonferroni correction; $p<0.05$ ). B, Population PSTH curve of the response to stimulus 1 when it was first in its sequence compared to population PSTH curve of the response to stimulus 1 when it was last in its sequence. The abscissa shows the time relative to the onset of stimulation. C, Same as in $\boldsymbol{B}$ but showing responses to stimulus 2. D, A scatter plot showing the distribution of stimulus indices of stimulus 2 versus stimulus 1 . Blue dots represent results from multiunit recordings and gray dots from single-unit recordings. The dashed lines mark the quadrant in which both indices are positive. $\boldsymbol{E}$, The distribution of neural indices from all tests.

less phasic compared to the other nuclei (compare PSTHs in Fig. 3). Nevertheless, the adaptation in the E was enhanced compared to all other nuclei (an amplitude attenuation of $\sim 75 \%$ in the $\mathrm{E}$ compared to $\sim 28 \%$ in the OT). Significant but mild adaptation at an ISI of $10 \mathrm{~s}$ was also revealed in the population of recording sites in the ICX (green histogram, $n=14$ ), a midbrain nucleus that provides the main source of auditory inputs to the OT (Knudsen, 1983).

To further study the long-lasting adaptation in the E, we measured adaptation profiles at ISIs of 30 and $60 \mathrm{~s}$. Figure $4 A$ shows an example of a multiunit recording from a single site in the E. In this example, sequences of five auditory stimuli with an ISI of $30 \mathrm{~s}$ were presented ( $2.5 \mathrm{~min}$ gap between sequences). The responses to the first stimulus in the sequence (upper raster plot) were much stronger than the responses to the last stimulus (lower raster plot). As in the OT, significant adaptation was observed at ISIs of 30 and $60 \mathrm{~s}$ (Fig. $4 B, C$ ). Moreover, the strength of adaptation in the E was enhanced compared to the OT. At 60 s ISI, the attenuation of the responses to subsequent stimuli was $56 \%$ in the $\mathrm{E}$ and $25 \%$ in the OT.
Specificity of long-lasting adaptation

To assess whether the long-lasting adaptation in the OT is stimulus specific, we tested the neurons with an alternating sequence composed of two narrowband stimuli ( $1 \mathrm{kHz}$ width) that differed in their central frequencies by a gap of $2 \mathrm{kHz}$. The central frequencies were selected to be equally distant from both sides to the best frequency. This selection procedure ensured that both stimuli were within the response range of the neuron (Reches and Gutfreund, 2008). The ISI was maintained at $10 \mathrm{~s}$ throughout the test. The first stimulus was repeated 10 times followed by 10 repetitions of the second stimulus, and so on, alternating between the two. In SSA, we expect the responses to the first presentation of a stimulus in each subsequence of 10 identical stimuli (the novel stimulus) to be stronger than the last. Figure $5 \mathrm{~A}$ shows the population response $(n=22)$ to the sequence of one stimulus (in blue) and to the sequence of the other stimulus (in red). Note that this is a cyclical stimulation paradigm; thus, the blue sequence arrives after the red sequence and the red sequence arrives after the blue. The average response to the first appearance of the stimulus was significantly stronger than the average response to all subsequent stimuli, for both frequencies ( $t$ test with Holm-Bonferroni correction, $p<0.05$ ). This indicates that the long-lasting adaptation in the OT is specific to the frequency of the stimulus. The stimuli adapted rapidly, reaching a significant reduction following one novel stimulus. The average PSTHs of the responses to the two stimuli when they are novel are compared in Figure 5, $B$ and $C$, with the average PSTHs of the responses to the same stimuli when they are last in the sequence. The adaptation pattern in this paradigm is comparable in magnitude with the pattern observed in the corresponding simple adaptation paradigm (Fig. 3, purple histogram); 30\% attenuation of the response in the simple adaptation paradigm compared to $\sim 35 \%$ in the alternating paradigm. This suggests that the adaptation is fully specific without cross-stimulus adaptation, at least under the tested conditions.

To examine the consistency of this effect, we calculated two SIs and a single neuron index (NI) for each site (see Materials and Methods). The scatter plot of the SIs of stimulus 1 versus SIs of stimulus 2 is shown in Figure 5D. A point appearing in the first quadrant (within the two dotted lines) implies stronger responses to the novel appearance of both stimuli. However, any point above the diagonal line implies SSA (Ulanovsky et al., 2003). The majority of points were within the first quadrant and only two points out of the 22 recording sites were below the diagonal line. Note that no apparent difference exists between distributions of the single-unit and multiunit recordings. The distribution of the neural indices is presented in Figure $5 E$. Evidently, the distribution is shifted to positive values (sign 


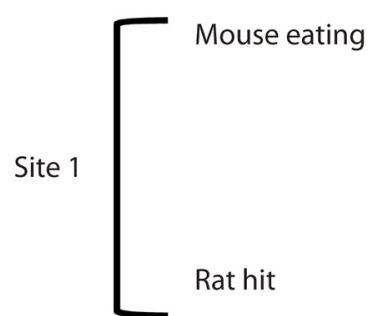
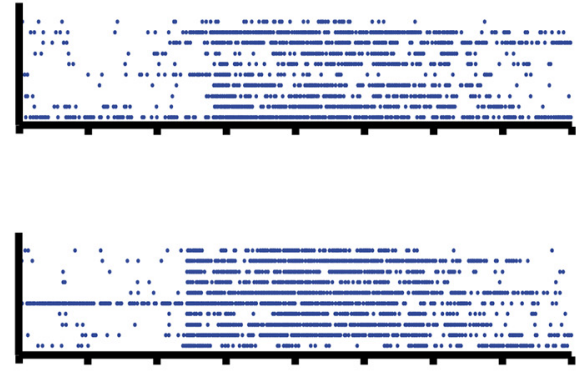

Mice sound
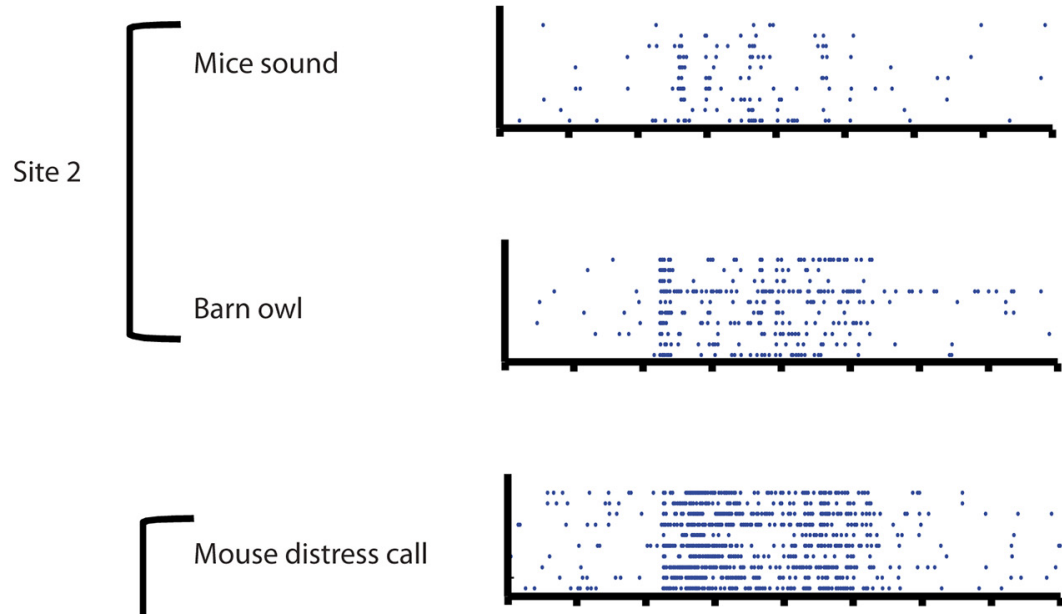

Mouse distress call

Site 3

Mouse scream
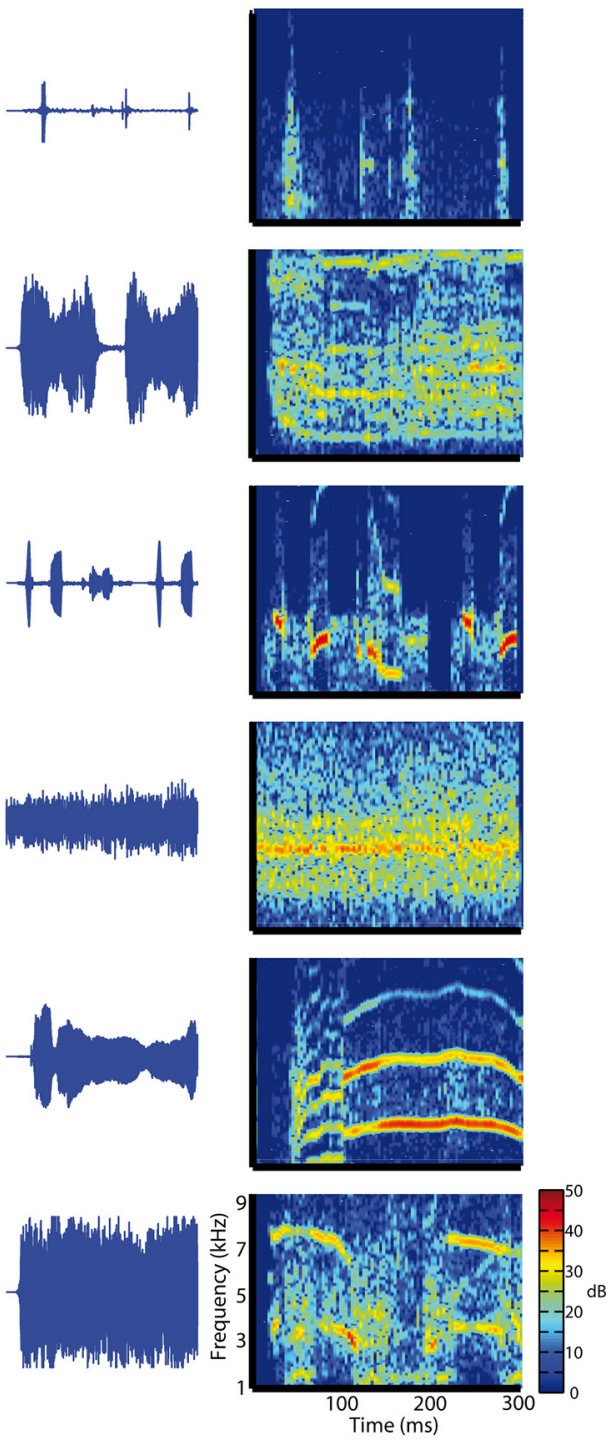

Figure 6. Neural responses, spectrograms and waveforms of the natural sounds used in this study. The raster plots on the left show neural responses from three tectal sites to the six sounds. Each raster plot show responses to 10 repetitions of the sound whose waveform and spectrogram are shown in the corresponding middle and right columns respectively. The upper two rasters show responses from one recording site, the middle two rasters from a different recording site, and the lower two rasters from a third recording site.

test, $p<0.001$ ), indicating a clear population tendency to respond stronger to novel stimuli.

In a different paradigm, to check the specificity of the adaptation to complex sounds, we used recordings of natural sounds. In each recording site, two sounds were drawn randomly from a library of six sounds. Tectal neurons responded well to all six sounds presented within their receptive fields (Fig. 6). A sequence of eight stimuli was presented with an ISI of $13 \mathrm{~s}$. The intersequence interval (time from last stimulus in a sequence to the first stimulus in the next sequence) was $73 \mathrm{~s}$. The first seven stimuli were identical and the eighth stimulus was different. If the adaptation is specific to the stimulus, we expect to regain the response to the last odd stimulus. As expected, the population responses to stimuli 2-7 were reduced compared to the response to the first stimulus ( $t$ test, Holm-Bonferroni correction, $p<0.05)$. However, the population neural response to the odd stimulus (red bar and red curve in Fig. $7 A, B$, respectively) was significantly larger than all the responses to stimuli $2-7$ ( $t$ test, Holm-Bonferroni correction, $p<0.05)$. Interest- ingly, the population response to the odd stimulus was also significantly larger than the population response to the first stimulus in the sequence ( $t$ test, $p<0.05$ ).

To further assess whether the adaptation is specific even at longer ISIs, we recorded the responses of neurons in the OT to sequences of stimuli with an ISI of $60 \mathrm{~s}$. The intersequence interval in this case was $5 \mathrm{~min}$. To minimize the length of the test, which can become overly long with such ISIs, we used here a sequence of three stimuli, the first two of which were identical and the last was different. The sequence was repeated 11 times. In each test, again, the two types of stimuli were drawn randomly from the same library of natural sounds. The population response $(n=12)$ to the second stimulus was reduced significantly compared to the first (Fig. 7C). On the other hand, the response to the last, odd stimulus was significantly stronger than the response to the second stimulus and not significantly different from the response to the first stimulus ( $t$ test, $p<0.05$ ). This demonstrates SSA to the type of stimulus at an ISI of $60 \mathrm{~s}$. The observation that the response to the odd stimulus was no less than the 
$|S|=13 \mathrm{sec}$
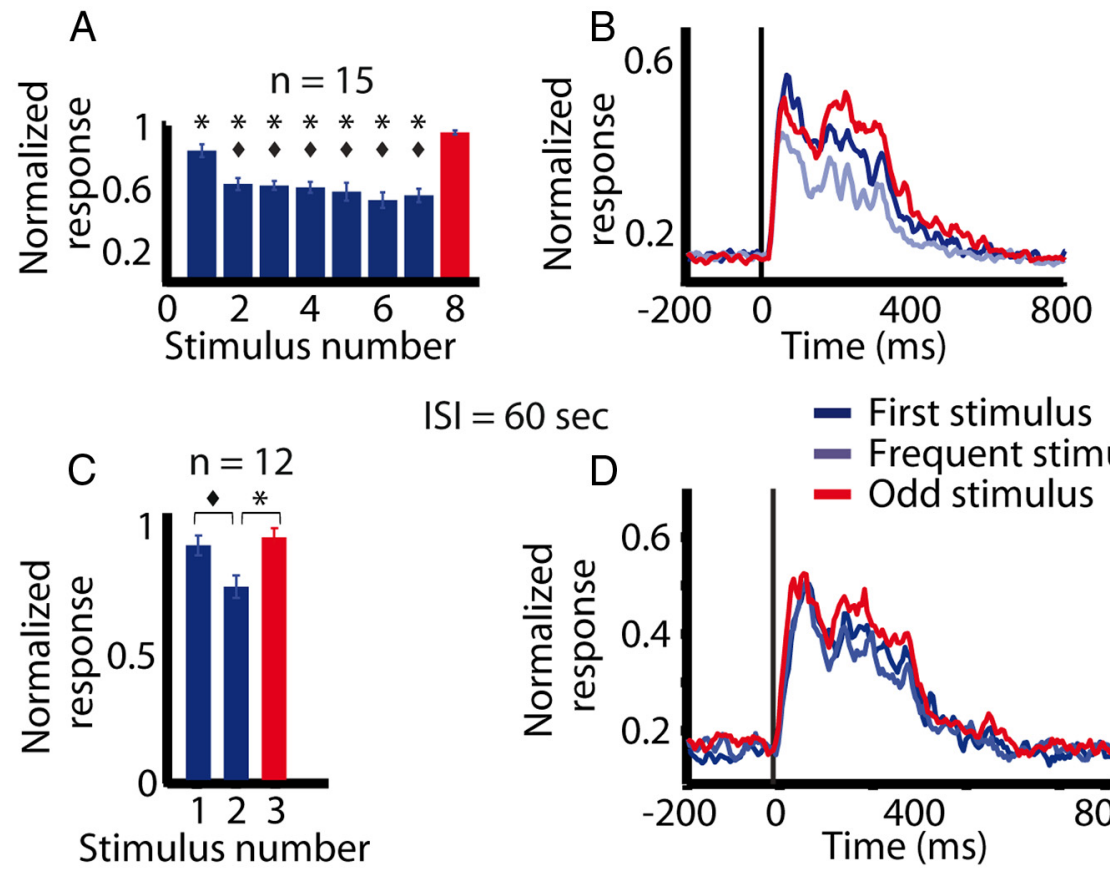

$|S|=60 \mathrm{sec}$
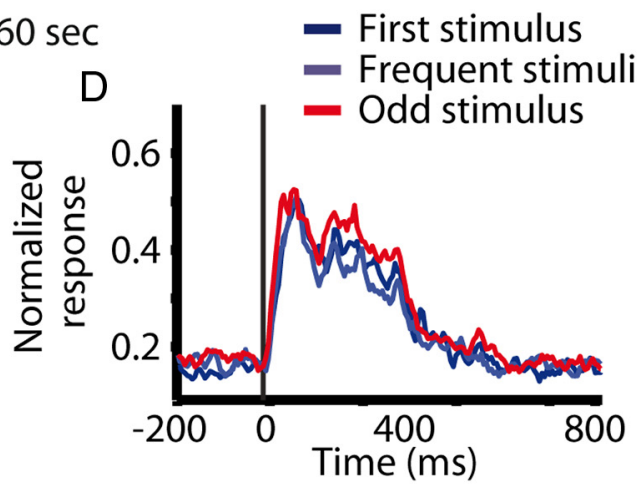

Figure 7. Adaptation in the OT to playback of natural sounds. $\boldsymbol{A}$, The population response as a function of the position of the stimulus in the sequence (ISI = $13 \mathrm{~s}$ ). The blue bars designate the responses to the seven repeated presentations of the standard stimulus and the red bar designates the response to the last odd stimulus. Error bars indicate SEMs. Diamonds indicate responses that were significantly smaller than the response to the first stimulus ( $t$ test, Holm-Bonferroni correction; $p<0.05$ ). Asterisks indicate responses that were significantly smaller than the response to the last odd stimulus ( $t$ test, Holm-Bonferroni correction; $p<0.05$ ). $\boldsymbol{B}$, The population PSTH curves of the response to the first stimulus (blue curve) compared to the average population PSTH curves of the responses to stimuli 5-7 (purple curve) and the population PSTH curve of the response to the last odd stimulus (red curve). C, The population response as a function of the position of the stimulus in the sequence (ISI $=60 \mathrm{~s}$ ). The blue bars designate the average responses to the first two identical stimuli and the red bar designates the average response to the third odd stimulus. $\boldsymbol{D}$, The population PSTH curves. The format is as in $\boldsymbol{B}$.

response to the first stimulus, again, suggests that there is no cross-stimulus adaptation at these relatively long ISIs.

\section{Behavioral habituation of the orienting reflex}

One caveat of correlating the neural adaptation with behavioral habituation is that the neural recordings are done under the influence of $\mathrm{N}_{2} \mathrm{O}$ (see Materials and Methods), while most previous behavioral studies of habituation were not (Weinberger et al., 1975; Valentinuzzi and Ferrari, 1997; Bala and Takahashi, 2000; Zimmer, 2006). Addressing this caveat, we have previously shown in barn owls that the pupil dilation reflex is maintained under the same levels of $\mathrm{N}_{2} \mathrm{O}$ used here, and moreover, the reflex undergoes habituation to short auditory stimuli $(300 \mathrm{~ms}$ ) presented at an ISI of $12 \mathrm{~s}$ (Netser et al., 2010). To extend this previous result to a different behavior, and to strengthen the comparison between the physiological results of this study and behavioral habituation, we characterized habituation of reflexive eye movements. In barn owls, eye movements are limited to $\pm 3^{\circ}$ (du Lac and Knudsen, 1990). The role in vision, if any, of these small movements is unknown. Moreover, it is unknown whether the eyes orient in a reflexive manner to auditory stimuli. To examine this, owls were positioned in the stereotaxic apparatus and anesthetized in the same way as for the physiological experiments. Horizontal eye movements were detected based on tracking the pecten oculi edge (arrow in Fig. $8 \mathrm{~A}$ inset), an apparent retinal landmark in barn owls (Netser et al., 2010). Two speakers positioned $1 \mathrm{~m}$ from the owl were used, one speaker at an azi- muth of $80^{\circ}$ to the right and another at an azimuth of $80^{\circ}$ to the left. In each trial, a sequence of six repetitions of a broadband noise with $300 \mathrm{~ms}$ duration was presented from one of the speakers. The ISI was $13 \mathrm{~s}$. The experiment was performed 30 times in each speaker, in four different owls. The average normalized eye responses from all the experiments in which the stimuli were presented on the right are shown in Figure $8 \mathrm{~A}$. The first event in the sequence tended to induce a stronger positive response, indicating a rightwards shift of the eye. This respond clearly habituated to the subsequent auditory stimuli. When the sound was generated from the left speaker the response to the first event was negative, indicating leftward motion, and similarly habituated to subsequent stimuli (Fig. $8 B)$. Thus, we show for the first time that the eyes of barn owls reflexively respond to a novel sound by an orienting movement toward its direction. Moreover, this behavioral response is maintained under the low levels of $\mathrm{N}_{2} \mathrm{O}$ used in the physiological experiments.

To examine the stimulus specificity of the orienting eye reflex, we used the same natural sounds used in the physiological experiments (Fig. 6). Sounds were delivered through earphones with a positive ITD value of $+80 \mu$ s (right ear leading) at the same sound intensity used in the physiological experiments. In each test, eight stimuli were presented with an ISI of $13 \mathrm{~s}$. The first seven stimuli were identical and the eighth stimulus was different. The types of stimuli were randomly chosen in different tests, as was done in the physiological experiments. The orienting response habituated to repeated stimuli. The average eye response to the first stimulus was significantly larger from the average eye responses to stimuli 2-7 ( $t$ test, Holm-Bonferroni correction, $p<0.05$ ). The eye average response to the odd stimulus (red bar in Fig. $8 C$ ) was significantly larger than the eye average responses to stimuli 3-7 ( $t$ test, HolmBonferroni correction $p<0.05$ ). Thus, the habituation was specific to the type of stimulus. The pattern of behavioral habituation resembled qualitatively the pattern of neural adaptation (compare Fig. $8 C$ with Fig. $7 A$ ). However, unlike the neural response, the average behavioral response to the last odd stimulus was not significantly above the response to the first stimulus.

\section{Discussion}

\section{Comparison with other types of adaptation}

SSA has been previously shown in various forms in the auditory system (Nelken and Ulanovsky, 2007; Reches and Gutfreund, 2008; Anderson et al., 2009; Malmierca et al., 2009), but the SSA we are describing here differs in two important aspects. First, most previous forms of SSA have been reported along the primary auditory pathway [inferior colliculus (Pérez-González et al., 2005), the medial geniculate body (Anderson et al., 2009), and the auditory cortex (Ulanovsky et al., 2003; Szymanski et al., 2009)], whereas this form was observed exclusively in the auditory localization pathway (OT) and a related forebrain structure 


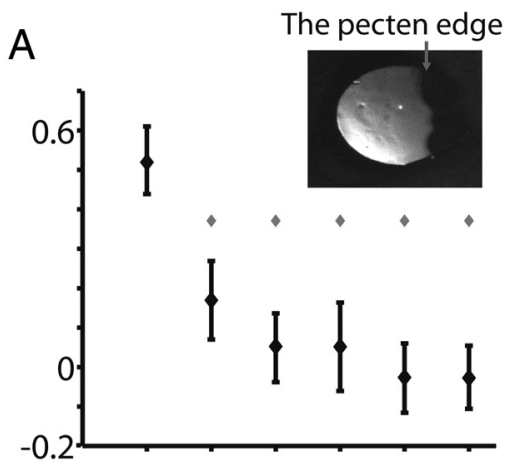

B

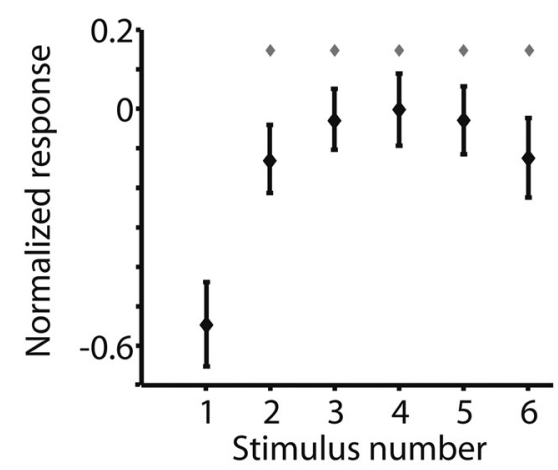

C

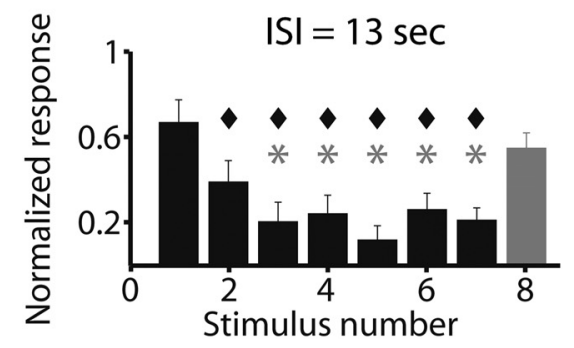

Figure 8. Habituation of reflexive eye movements. $A$, The population pecten responses of the right eye to a sequence of six auditory stimuli presented, with an $|S|$ of $13 \mathrm{~s}$, from a speaker on the right side. The error bars designate SEMs. The gray diamonds indicate responses that were significantly smaller than the response to the first stimulus in the sequence $(t$ test, $\mathrm{Holm}-$ Bonferroni correction; $p<0.05$ ). The inset displays a video frame showing the infrared light reflected from the right eye. The edge of the pecten oculi is marked by the arrow. $\boldsymbol{B}$, The population pecten responses of the right eye to a sequence of six auditory stimuli presented, with an ISI of $13 \mathrm{~s}$, from a speaker on the left side. The format is as in $A$. C, The population pecten responses of the right eye to a sequence of eight auditory stimuli of which the eighth stimulus was different. The black bars indicate the responses to the seven repeated presentations of the same standard stimulus. The gray bar shows the average response to the last odd stimulus. Auditory stimuli were presented through earphones with an ITD of $+80 \mu$ s (right ear leading). Error bars indicate SEMs. Diamonds indicate responses that were significantly smaller than the response to the first stimulus ( $t$ test, Holm-Bonferroni correction; $p<0.05$ ). Asterisks indicate responses that were significantly smaller than the response to the last odd stimulus.

(E). Second, previous forms of SSA have been reported to diminish at ISIs longer than $2 \mathrm{~s}$ (Ulanovsky et al., 2003) or have only been studied at ISIs $<2 \mathrm{~s}$ (Reches and Gutfreund, 2008; von der Behrens et al., 2009; Antunes et al., 2010), whereas the adaptation identified here is active and specific in ISIs as long as $60 \mathrm{~s}$. To the best of our knowledge, a comparable adaptation has not been reported in the auditory system.

Several previous studies reported long-lasting effects of adaptation in the auditory cortex in the time range of tens of seconds to minutes (Weinberger et al., 1975; Condon and Weinberger,
1991; Ulanovsky et al., 2004). However, adaptations were induced by long periods of stimulation in these studies. For example, Condon and Weinberger (1991) used a $1.25 \mathrm{~Hz}$ train of $300-500$ stimuli (300 ms duration each) to induce specific adaptation that lasted for several minutes. The above-mentioned studies are in agreement with the notion that the dynamics of neural adaptation complies with stimulus duration (Marom, 2010): a short-duration stimulus is expected to induce shortlasting adaptation, and vice versa (Varela et al., 1997; Ulanovsky et al., 2004). This concept is not in line with the adaptation reported here whereby a relatively short stimulus was sufficient to induce long-lasting adaptation of $1 \mathrm{~min}$ and possibly more. Therefore, the SSA identified here differs from other known forms of auditory SSA, and is likely to involve different mechanisms and play different roles in auditory perception.

The duration of the memory trace of neural adaptation has direct implications on the behaviors that it can support. In many instances, habituation of behavioral responses has been demonstrated with short stimuli that were separated by intervals of several seconds to minutes (Thompson and Spencer, 1966; Weinberger et al., 1975; Bala and Takahashi, 2000; Zimmer, 2006; Glanzman, 2009). Specifically in barn owls, rapid habituation of the pupil dilation response (PDR) was demonstrated using short, low-intensity auditory stimuli with ISIs of $10-13 \mathrm{~s}$. This habituation of the PDR was mediated by microstimulations in the OT (Netser et al., 2010) and was highly specific to the location or frequency of the stimulus (Bala and Takahashi, 2000; Bala et al., 2003; Spitzer et al., 2003; Bala et al., 2007). Therefore, the finding reported here that specific adaptation in the OT can reach time spans of tens of seconds suggests that this type of neural adaptation is a neural correlate of the behavioral habituation. This hypothesis is strengthened by our demonstration of behavioral habituation of the eye-orienting reflex to the same stimulus and at the same experimental conditions in which long-lasting neural adaptation was measured, thus ruling out possible effects of anesthesia or of specific stimulus conditions. The general pattern of behavioral habituation (Fig. $8 C$ ) resembled the pattern of neural adaptation at comparable ISIs (Fig. 7A). Both behavioral and neural responses habituated, and in both, the responses to the odd stimulus were elevated. However, the neural responses to the odd stimulus tended to exceed the neural responses to the first stimulus, while the behavioral responses did not. This difference may indicate that there is no simple linear relationship between the neural responses and the behavior and that other brain regions may also influence the final behavioral response.

\section{Origin of adaptation}

The ICX provides the direct auditory input to the OT (Knudsen, 1983). We report small but significant adaptation at $10 \mathrm{~s}$ intervals already in the ICX. The same stimulus protocol induced stronger adaptation in the OT and even stronger adaptation in the E. In barn owls, auditory information from the OT travels to the forebrain E through the tectofugal pathway (Reches and Gutfreund, 2009). Therefore, the simplistic interpretation would be that the adaptation originates in the ICX and is then strengthened by further processing in the ascending pathway. However, this interpretation is complicated by the fact that there are multiple loops. The OT projects back to the ICX (Hyde and Knudsen, 2000; Luksch et al., 2000; Gutfreund et al., 2002). The E is likely to form a loop with the optic tectum through the auditory arcopallium (Knudsen et al., 1995; Shimizu and Bowers, 1999), which also sends projections to the ICX (Knudsen et al., 1995). Given these multiple loops, the data in Figure 3 cannot resolve the origin 
of adaptation. It is possible that the adaptation originates in the OT and projects back to the ICX. Another possibility is that it originates in forebrain networks, giving rise to top-down signals that enhance tectal responses to novel stimuli.

\section{Mechanisms of adaptation}

One common model to explain SSA is that the two stimuli activate separate paths to the recorded neuron and that basic adaptation mechanisms (synaptic depression or intrinsic cellular mechanisms) act at levels where the activation is separated (Eytan et al., 2003). An intriguing observation in our study was that the responses to the last odd stimuli were not weaker than the responses to the first stimuli (Fig. 7), suggesting a complete lack of cross-stimulus adaptation at these long ISIs. This implies, according to the model, that the basic adaptation processes must take place at levels where there is no overlap between the neural elements carrying the signals from the two stimuli. Since the frequencies of the natural sounds used in these experiments overlapped substantially (Fig. 6), the results are unlikely to be accounted for only by basic response suppressions at lower, frequency-specific levels. To compute the novelty of complex broadband sounds, a network is required that compares the neural responses to the current stimulus with previous responses based on an integration of information about frequency and amplitude modulation. Integration of high-level acoustic features has been shown to occur at forebrain auditory areas in mammals and songbirds (Doupe, 1997; Romanski and Goldman-Rakic, 2002; Lehongre and Del Negro, 2011). Therefore, it is plausible that circuits in the forebrain compute the novelty of the incoming sound and modulate tectal responses accordingly. In this respect, forebrain-tectal connections have been shown to be able to modulate tectal responses (Winkowski and Knudsen, 2006; Winkowski and Knudsen, 2008).

\section{The gaze control system and habituation}

Regardless of the specific mechanism for generating adaptation, a clear distinction can be made between the tectofugal pathway and the thalamofugal pathway. Significant adaptation was not found in $\mathrm{nOv}$, the primary thalamic auditory nucleus, and the first station in the thalamofugal pathway (Proctor and Konishi, 1997). The avian $\mathrm{nOv}$ is anatomically divided into shell and core (Durand et al., 1992). The functional significance of this division is unknown. Electrolytic lesions performed at the end of our last experiment were found to be well within the core. Since the core in barn owls is by far larger compared to shell regions (Pérez et al., 2009), it is likely that most of our recording sites in nOv were from the core region. Therefore, we cannot rule out the possibility that long-lasting adaptation of neural responses exits in the lateral or medial shell of nOv. Related to this, recent studies in rodents reported robust SSA in the medial subdivision of the primary auditory thalamus (Anderson et al., 2009; Antunes et al., 2010). However, this thalamic SSA recorded was relatively short latency (up to $2 \mathrm{~s}$ ), and therefore the lack of long-lasting adaptation in $\mathrm{nOv}$ is not in conflict with the findings in mammals.

Recent studies suggest that the avian tectofugal pathway carries information about the saliency of the stimulus from the OT to the forebrain (Marín et al., 2007; Reches and Gutfreund, 2009; Reches et al., 2010). Moreover, the tectofugal pathway is considered to be homologous to the mammalian colliculo-pulvinarcortical pathway (Karten, 1969; Karten and Shimizu, 1989), which has been suggested to play an important role in the coordination of spatial attention between the SC and the cortex (Robinson and Petersen, 1992; Olshausen et al., 1993; Shipp, 2004).
Why it is that adaptation is pronounced specifically in structures related to gaze control and attention (OT and E)?

Substantial evidence supports the notion that the OT (and its mammalian homolog, the superior colliculus) contains the circuitry to represent the saliency of the incoming stimuli, a prerequisite for selective attention (Ingle, 1975; Frost et al., 1981; Basso and Wurtz, 1997; McPeek and Keller, 2002; Khanbabaie et al., 2007; Boehnke and Munoz, 2008; Mysore et al., 2010). An important element in stimulus saliency is its history. Stimuli that differ from previous stimuli (i.e., surprising stimuli) are perceived as being more salient (Posner, 1981; Itti and Koch, 2000). Therefore, we propose that specific adaptation is pronounced in the OT as part of its role in stimulus selection, giving rise to an appealing hypothesis that the neural mechanisms for highlighting a salient stimulus in space overlap with the mechanisms for highlighting a salient stimulus in time.

\section{References}

Anderson LA, Christianson GB, Linden JF (2009) Stimulus-specific adaptation occurs in the auditory thalamus. J Neurosci 29:7359-7363.

Antunes FM, Nelken I, Covey E, Malmierca MS (2010) Stimulus-specific adaptation in the auditory thalamus of the anesthetized rat. PLoS One 5:e14071.

Arthur BJ (2005) Distribution within the barn owl's inferior colliculus of neurons projecting to the optic tectum and thalamus. J Comp Neurol 492:110-121.

Bala AD, Takahashi TT (2000) Pupillary dilation response as an indicator of auditory discrimination in the barn owl. J Comp Physiol A 186:425-434.

Bala AD, Spitzer MW, Takahashi TT (2003) Prediction of auditory spatial acuity from neural images on the owl's auditory space map. Nature 424:771-774.

Bala AD, Spitzer MW, Takahashi TT (2007) Auditory spatial acuity approximates the resolving power of space-specific neurons. PLoS ONE 2:e675.

Barry RJ (2009) Habituation of the orienting reflex and the development of preliminary process theory. Neurobiol Learn Mem 92:235-242.

Basso MA, Wurtz RH (1997) Modulation of neuronal activity by target uncertainty. Nature 389:66-69.

Benowitz LI, Karten HJ (1976) Organization of the tectofugal visual pathway in the pigeon: a retrograde transport study. J Comp Neurol 167:503-520.

Boehnke SE, Munoz DP (2008) On the importance of the transient visual response in the superior colliculus. Curr Opin Neurobiol 18:544-551.

Brainard MS, Knudsen EI (1993) Experience-dependent plasticity in the inferior colliculus: a site for visual calibration of the neural representation of auditory space in the barn owl. J Neurosci 13:4589-4608.

Condon CD, Weinberger NM (1991) Habituation produces frequencyspecific plasticity of receptive fields in the auditory cortex. Behav Neurosci 105:416-430.

Dong S, Clayton DF (2009) Habituation in songbirds. Neurobiol Learn Mem 92:183-188.

Doupe AJ (1997) Song- and order-selective neurons in the songbird anterior forebrain and their emergence during vocal development. J Neurosci 17:1147-1167.

du Lac S, Knudsen EI (1990) Neural maps of head movement vector and speed in the optic tectum of the barn owl. J Neurophysiol 63:131-146.

Durand SE, Tepper JM, Cheng MF (1992) The shell region of the nucleus ovoidalis: a subdivision of the avian auditory thalamus. J Comp Neurol 323:495-518.

Eytan D, Brenner N, Marom S (2003) Selective adaptation in networks of cortical neurons. J Neurosci 23:9349-9356.

Farley BJ, Quirk MC, Doherty JJ, Christian EP (2010) Stimulus-specific adaptation in auditory cortex is an NMDA-independent process distinct from the sensory novelty encoded by the mismatch negativity. J Neurosci 30:16475-16484.

Frost BJ, Scilley PL, Wong SC (1981) Moving background patterns reveal double-opponency of directionally specific pigeon tectal neurons. Exp Brain Res 43:173-185.

Glanzman DL (2009) Habituation in Aplysia: the Cheshire cat of neurobiology. Neurobiol Learn Mem 92:147-154. 
Gutfreund Y, Zheng W, Knudsen EI (2002) Gated visual input to the central auditory system. Science 297:1556-1559.

Holm S (1979) A simple sequentially rejective multiple test procedure. Scand J Statist 6:65-70.

Hyde PS, Knudsen EI (2000) Topographic projection from the optic tectum to the auditory space map in the inferior colliculus of the barn owl. J Comp Neurol 421:146-160.

Ingle D (1975) Focal attention in the frog: behavioral and physiological correlates. Science 188:1033-1035.

Itti L, Koch C (2000) A saliency-based search mechanism for overt and covert shifts of visual attention. Vision Res 40:1489-1506.

Karten HJ (1969) Organization of avian telencephalon and some speculations on phylogeny of amniote telencephalon. Ann NY Acad Sci 167:164-179.

Karten HJ, Shimizu T (1989) The origins of neocortex: connections and lamination as distinct events in evolution. J Cogn Neurosci 1:290-301.

Katz Y, Heiss JE, Lampl I (2006) Cross-whisker adaptation of neurons in the rat barrel cortex. J Neurosci 26:13363-13372.

Khanbabaie R, Mahani AS, Wessel R (2007) Contextual interaction of GABAergic circuitry with dynamic synapses. J Neurophysiol 97:28022811.

Knudsen EI (1982) Auditory and visual maps of space in the optic tectum of the owl. J Neurosci 2:1177-1194.

Knudsen EI (1983) Subdivisions of the inferior colliculus in the barn owl (Tyto alba). J Comp Neurol 218:174-186.

Knudsen EI, Cohen YE, Masino T (1995) Characterization of a forebrain gaze field in the archistriatum of the barn owl: microstimulation and anatomical connections. J Neurosci 15:5139-5151.

Lehongre K, Del Negro C (2011) Representation of the bird's own song in the canary HVC: contribution of broadly tuned neurons. Neuroscience 173:93-109.

Luksch H, Gauger B, Wagner H (2000) A candidate pathway for a visual instructional signal to the barn owl's auditory system. J Neurosci 20:RC70.

Malmierca MS, Cristaudo S, Pérez-González D, Covey E (2009) Stimulusspecific adaptation in the inferior colliculus of the anesthetized rat. J Neurosci 29:5483-5493.

Marín G, Salas C, Sentis E, Rojas X, Letelier JC, Mpodozis J (2007) A cholinergic gating mechanism controlled by competitive interactions in the optic tectum of the pigeon. J Neurosci 27:8112-8121.

Marom S (2010) Neural timescales or lack thereof. Prog Neurobiol 90:16-28.

McPeek RM, Keller EL (2002) Saccade target selection in the superior colliculus during a visual search task. J Neurophysiol 88:2019-2034.

Miller GL, Knudsen EI (2003) Adaptive plasticity in the auditory thalamus of juvenile barn owls. J Neurosci 23:1059-1065.

Mysore SP, Asadollahi A, Knudsen EI (2010) Global inhibition and stimulus competition in the owl optic tectum. J Neurosci 30:1727-1738.

Nelken I, Ulanovsky N (2007) Mismatch negativity and stimulus-specific adaptation in animal models. J Psychophysiol 21:214-223.

Netser S, Ohayon S, Gutfreund Y (2010) Multiple manifestations of microstimulation in the optic tectum: eye movements, pupil dilations, and sensory priming. J Neurophysiol 104:108-118.

Olshausen BA, Anderson CH, Van Essen DC (1993) A neurobiological model of visual attention and invariant pattern recognition based on dynamic routing of information. J Neurosci 13:4700-4719.

Pérez ML, Peña JL (2006) Comparison of midbrain and thalamic spacespecific neurons in barn owls. J Neurophysiol 95:783-790.

Pérez ML, Shanbhag SJ, Peña JL (2009) Auditory spatial tuning at the crossroads of the midbrain and forebrain. J Neurophysiol 102:1472-1482.

Pérez-González D, Malmierca MS, Covey E (2005) Novelty detector neurons in the mammalian auditory midbrain. Eur J Neurosci 22:2879-2885.

Posner MI (1981) Cognition and neural systems. Cognition 10:261-266.

Proctor L, Konishi M (1997) Representation of sound localization cues in the auditory thalamus of the barn owl. Proc Natl Acad Sci U S A 94:10421-10425.

Rankin CH, Abrams T, Barry RJ, Bhatnagar S, Clayton DF, Colombo J, Coppola G, Geyer MA, Glanzman DL, Marsland S, McSweeney FK, Wilson DA, Wu CF, Thompson RF (2009) Habituation revisited: an updated and revised description of the behavioral characteristics of habituation. Neurobiol Learn Mem 92:135-138.

Reches A, Gutfreund Y (2008) Stimulus-specific adaptations in the gaze control system of the barn owl. J Neurosci 28:1523-1533.

Reches A, Gutfreund Y (2009) Auditory and multisensory responses in the tectofugal pathway of the barn owl. J Neurosci 29:9602-9613.

Reches A, Netser S, Gutfreund Y (2010) Interactions between stimulusspecific adaptation and visual auditory integration in the forebrain of the barn owl. J Neurosci 30:6991-6998.

Robinson DL, Petersen SE (1992) The pulvinar and visual salience. Trends Neurosci 15:127-132.

Romanski LM, Goldman-Rakic PS (2002) An auditory domain in primate prefrontal cortex. Nat Neurosci 5:15-16.

Shimizu T, Bowers AN (1999) Visual circuits of the avian telencephalon: evolutionary implications. Behav Brain Res 98:183-191.

Shipp S (2004) The brain circuitry of attention. Trends Cogn Sci 8:223-230.

Sobotka S, Ringo JL (1994) Stimulus specific adaptation in excited but not in inhibited cells in inferotemporal cortex of macaque. Brain Res 646:95-99.

Spitzer MW, Bala AD, Takahashi TT (2003) Auditory spatial discrimination by barn owls in simulated echoic conditions. J Acoust Soc Am 113:1631-1645.

Szymanski FD, Garcia-Lazaro JA, Schnupp JW (2009) Current source density profiles of stimulus-specific adaptation in rat auditory cortex. J Neurophysiol 102:1483-1490.

Thompson RF (2009) Habituation: a history. Neurobiol Learn Mem 92:127-134

Thompson RF, Spencer WA (1966) Habituation: a model phenomenon for the study of neuronal substrates of behavior. Psychol Rev 73:16-43.

Ulanovsky N, Las L, Nelken I (2003) Processing of low-probability sounds by cortical neurons. Nat Neurosci 6:391-398.

Ulanovsky N, Las L, Farkas D, Nelken I (2004) Multiple time scales of adaptation in auditory cortex neurons. J Neurosci 24:10440-10453.

Valentinuzzi VS, Ferrari EA (1997) Habituation to sound during morning and night sessions in pigeons (Columba livia). Physiol Behav 62: 1203-1209.

Varela JA, Sen K, Gibson J, Fost J, Abbott LF, Nelson SB (1997) A quantitative description of short-term plasticity at excitatory synapses in layer $2 / 3$ of rat primary visual cortex. J Neurosci 17:7926-7940.

von der Behrens W, Bäuerle P, Kössl M, Gaese BH (2009) Correlating stimulus-specific adaptation of cortical neurons and local field potentials in the awake rat. J Neurosci 29:13837-13849.

Wagner H, Takahashi T, Konishi M (1987) Representation of interaural time difference in the central nucleus of the barn owl's inferior colliculus. J Neurosci 7:3105-3116.

Wathey JC, Pettigrew JD (1989) Quantitative analysis of the retinal ganglion cell layer and optic nerve of the barn owl Tyto alba. Brain Behav Evol 33:279-292.

Weinberger NM, Oleson TD, Ashe JH (1975) Sensory system neural activity during habituation of the pupillary orienting reflex. Behav Biol 15:283-301.

Winkowski DE, Knudsen EI (2006) Top-down gain control of the auditory space map by gaze control circuitry in the barn owl. Nature 439:336-339.

Winkowski DE, Knudsen EI (2008) Distinct mechanisms for top-down control of neural gain and sensitivity in the owl optic tectum. Neuron 60:698-708.

Zimmer H (2006) Habituation of the orienting response as reflected by the skin conductance response and by endogenous event-related brain potentials. Int J Psychophysiol 60:44-58. 